\title{
Association between Plasma Levels of Growth Differentiation Factor-15 and Renal Function in the Elderly: Korean Frailty and Aging Cohort Study
}

\author{
Jin Sug Kim ${ }^{a}$ Sunyoung Kim ${ }^{b}$ Chang Won Won ${ }^{b}$ Kyung Hwan Jeong ${ }^{a}$ \\ ${ }^{a}$ Division of Nephrology, Department of Internal Medicine, College of Medicine, Kyung Hee \\ University, Seoul, Republic of Korea; b Department of Family Medicine, College of Medicine, \\ Kyung Hee University, Seoul, Republic of Korea
}

\section{Keywords}

Growth differentiation factor-15 - Renal function · Elderly

\begin{abstract}
Background/Aims: Growth differentiation factor-15 (GDF-15) expression has been reported to increase in response to tissue damage and has recently emerged as a useful biomarker for various diseases. Although emerging evidence supports the clinicopathological value of GDF15 in renal impairment, few studies have analyzed it in the elderly. Thus, we conducted a crosssectional study to investigate the association of plasma GDF-15 with renal function and the presence of chronic kidney disease (CKD) in community-dwelling elderly. Materials: The present study was based on the baseline data of the Korean Frailty and Aging Cohort Study (KFACS), a nationwide cohort study that began in 2016. Of the 1,559 participants assessed in the first year, 443 with available plasma GDF-15 data were enrolled in this study. We investigated the association of plasma GDF-15 levels with clinical and biochemical parameters. The study population was divided into two groups according to renal function (CKD and non-CKD groups) to investigate whether GDF-15 can determine the presence of renal dysfunction in the elderly. Plasma GDF-15 was measured by enzyme-linked immunosorbent assay (ELISA) kit. Results: In a simple regression analysis, the levels of plasma GDF-15 were negatively correlated with estimated glomerular filtration rate (eGFR; $r=-0.383, p<0.001$ ). In multiple linear regression analysis, GDF-15 levels were still significantly correlated with eGFR, even after adjusting for other parameters $(r=-0.259, p<0.001)$. Plasma GDF-15 levels were significantly higher in the elderly with CKD than in those without CKD $(2,364.025 \pm 1,052.23 \mathrm{ng} / \mathrm{L}$ and $1,451.23 \pm 835.79 \mathrm{ng} / \mathrm{L}$, respectively; $p<0.001)$. The optimal cut-off value of plasma GDF-15 for detecting the presence of CKD was $1,699.4 \mathrm{ng} / \mathrm{L}$ ( $76.5 \%$ sensitivity and $76.0 \%$ specificity), as determined by the receiver operating characteristic curve. The area under the curve was
\end{abstract}




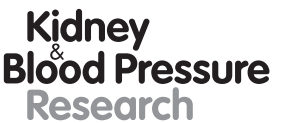

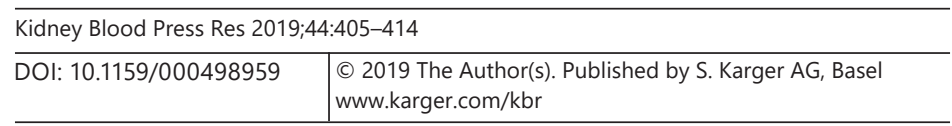

Kim et al.: Association between Plasma GDF-15 Levels and Renal Function in the Elderly

$0.793 \pm 0.033$ (95\% Cl 0.729-0.857, $p<0.001)$. Conclusion: Plasma GDF-15 levels were negatively associated with eGFR and were significantly increased in the elderly with CKD. Our results suggested that plasma GDF-15 might be a useful marker for discriminating renal impairment in the elderly. Further large and prospective outcome studies of extended duration are needed.

(C) 2019 The Author(s)

Published by S. Karger AG, Basel

\section{Introduction}

Renal dysfunction is a common clinical problem in the elderly [1]. Considering that the risk factors for renal impairment, such as diabetes mellitus (DM) and hypertension, are increasing with improved life expectancy, renal dysfunction in the elderly is expected to become more frequent [2]. Since renal dysfunction is associated with higher morbidity and mortality in the elderly [2, 3], further studies are needed to assess and diagnose it in this population. Although several parameters have been reported as biomarkers for estimating renal function, few studies have explored them in the elderly [4].

Growth differentiation factor-15 (GDF-15) is a member of the transforming growth factor $\beta$-superfamily and is widely expressed in adult tissues [5]. Elevated GDF-15 levels are associated with chronic inflammation, oxidative stress, and tissue injury [6]. Recently, GDF-15 has emerged as a biomarker for diagnosis and prediction of disease progression in various diseases, including cardiovascular and chronic inflammatory diseases [7]. Some studies have reported that higher GDF-15 levels are also associated with renal disorders such as diabetic nephropathy, idiopathic membranous nephropathy, and IgA nephropathy [8-11]. Other researchers have demonstrated that GDF-15 levels are correlated with prognosis of chronic kidney disease (CKD) $[12,13]$. However, only a few studies have analyzed the association between GDF-15 and renal function in the elderly [14]. To our knowledge, there has been no study suggesting the cut-off level of plasma GDF-15 for discrimination of CKD presence in the elderly.

Therefore, we conducted this study to determine the association between plasma GDF-15 level and renal function in elderly individuals. We evaluated the correlation between plasma GDF-15 level and estimated glomerular filtration rate (eGFR) and analyzed the level of plasma GDF-15 in the elderly with or without CKD. We also determined whether plasma GDF-15 level can discern the presence of CKD in the elderly and obtained the optimal cut-off level of plasma GDF-15. The first-year cross-sectional data from the nationwide Korean Frailty and Aging Cohort Study (KFACS) were used in this study.

\section{Methods}

\section{Study Population and Design}

The study population was derived from the KFACS, a nationwide, multicenter, longitudinal cohort study in which the baseline survey was conducted from 2016 to 2017. The participants were recruited from community-dwelling residents in urban and rural regions across the nation. Community residents with no plans to move in the next two years, aged 70-84 years, without serious problems in communication, and living independently at home were eligible for participation. Further details of the KFACS have been previously described [15]. Of the 1,559 participants who enrolled in the KFACS during the first year, 443 with available plasma GDF-15 data were included in this study. After investigating the association between 
Table 1. Clinical and biochemical characteristics of the study population

\begin{tabular}{lc}
\hline & Overall $(n=443)$ \\
\hline Age, years & $76.49 \pm 3.91$ \\
Gender & \\
$\quad$ Male & $229(51.7 \%)$ \\
$\quad$ Female & $214(48.3 \%)$ \\
BMI & $24.26 \pm 2.88$ \\
Hypertension, $n$ (\%) & $241(54.4 \%)$ \\
Dyslipidemia, $n$ (\%) & $132(29.8 \%)$ \\
Diabetes mellitus, $n(\%)$ & $88(19.9 \%)$ \\
Coronary artery disease, $n(\%)$ & $35(7.9 \%)$ \\
Cerebrovascular disease, $n(\%)$ & $16(3.7 \%)$ \\
Protein, g/dL & $7.01 \pm 0.38$ \\
Albumin, g/dL & $4.33 \pm 0.24$ \\
Creatinine, mg/dL & $0.84 \pm 0.25$ \\
eGFR, mL/min/1.73 m ${ }^{2}$ & $77.74 \pm 13.83$ \\
Glucose, mg/dL & $103.76 \pm 19.96$ \\
Calcium, mg/dL & $9.26 \pm 0.36$ \\
Phosphorus, mg/dL & $3.51 \pm 0.47$ \\
Sodium, mmol/L & $141.28 \pm 2.13$ \\
Potassium, mmol/L & $4.50 \pm 0.53$ \\
Total cholesterol, mg/dL & $185.25 \pm 37.48$ \\
HDL cholesterol, mg/dL & $52.09 \pm 13.52$ \\
LDL cholesterol, mg/dL & $110.16 \pm 33.38$ \\
Triglyceride, mg/dL & $115.02 \pm 51.41$ \\
Hemoglobin, g/dL & $13.38 \pm 1.44$ \\
25(OH) vit D, nmol/L & $21.38 \pm 8.48$ \\
hs-CRP, mg/dL & $1.29 \pm 1.60$ \\
GDF-15, ng/L & $1,556.32 \pm 910.09$ \\
Proteinuria, $n(\%)$ & $9(2.0 \%)$ \\
Hematuria, $n(\%)$ & $117(26.4 \%)$ \\
\hline & \\
\hline & \\
& \\
&
\end{tabular}

BMI, body mass index; eGFR, estimated glomerular filtration rate; HDL, high-density lipoprotein; LDL, low-density lipoprotein; $25(\mathrm{OH})$ vit D, 25-hydroxy vitamin D; hs-CRP, high-sensitivity C-reactive protein; GDF-15, growth differentiation factor-15.

GDF-15 and renal function in the whole study population, we divided the participants into two groups according to renal function (CKD and non-CKD) to analyze the diagnostic predictive value of GDF-15 levels for CKD.

All study procedures complied with the ethical guidelines of the 1975 Declaration of Helsinki, as revised in 2000. The study protocol was approved by the Institutional Review Board of Kyung Hee University Medical Center (IRB No. 2018-01-052). Written consent was obtained from all participants.

\section{Variables and Definitions}

Baseline variables included age, gender, body mass index (BMI), and underlying diseases such as hypertension, dyslipidemia, and DM. Laboratory findings, including serum protein, albumin, creatinine, glucose, electrolytes, lipid profiles, and inflammatory markers, were analyzed. Renal function was assessed via the eGFR, calculated using the Chronic Kidney Disease Epidemiology Collaboration (CKD-EPI) equation [16]. CKD was defined as eGFR $<60$ $\mathrm{mL} / \mathrm{min} / 1.73 \mathrm{~m}^{2}$. In this study, we defined proteinuria as $\geq 1+$ by the dipstick test, and hematuria as $\geq 3$ urine red blood cells per high-power field on a random urine sample. 
Table 2. Simple and multiple linear regression analyses of plasma GDF-15 level

\begin{tabular}{|c|c|c|c|c|c|c|c|c|}
\hline & \multicolumn{4}{|c|}{ Simple linear regression analysis } & \multicolumn{4}{|c|}{ Multiple linear regression analysis } \\
\hline & B & SE & $\beta$ & $p$ & B & SE & $\beta$ & $p$ \\
\hline Age (years) & 34.810 & 10.958 & 0.150 & 0.002 & 18.909 & 9.779 & 0.081 & 0.054 \\
\hline Male gender & -64.547 & 86.573 & -0.035 & 0.456 & & & & \\
\hline eGFR $\left(\mathrm{mL} / \mathrm{min} / 1.73 \mathrm{~m}^{2}\right)$ & -25.184 & 2.898 & -0.383 & $<0.001$ & -17.247 & 3.050 & -0.259 & $<0.001$ \\
\hline BMI & 2.219 & 15.042 & 0.007 & 0.883 & & & & \\
\hline Hypertension & 125.379 & 86.710 & 0.069 & 0.149 & & & & \\
\hline Dyslipidemia & 234.496 & 94.624 & 0.118 & 0.014 & -9.750 & 85.876 & -0.005 & 0.910 \\
\hline Diabetes mellitus & 891.217 & 99.560 & 0.393 & $<0.001$ & 813.064 & 113.447 & 0.357 & $<0.001$ \\
\hline Coronary artery disease & 317.351 & 159.765 & 0.094 & 0.048 & 176.215 & 135.370 & 0.053 & 0.194 \\
\hline Cerebrovascular disease & 17.975 & 232.783 & 0.004 & 0.938 & & & & \\
\hline Protein $(\mathrm{g} / \mathrm{dL})$ & 300.412 & 112.639 & 0.126 & 0.008 & 139.262 & 102.821 & 0.058 & 0.176 \\
\hline $\operatorname{Albumin}(\mathrm{g} / \mathrm{dL})$ & 228.163 & 178.281 & 0.061 & 0.201 & & & & \\
\hline Glucose (mg/dL) & 7.557 & 2.142 & 0.166 & $<0.001$ & -3.464 & 2.192 & -0.076 & 0.115 \\
\hline Calcium (mg/dL) & 267.412 & 119.128 & 0.106 & 0.025 & 133.221 & 112.175 & 0.053 & 0.236 \\
\hline Phosphorus (mg/dL) & 274.680 & 90.664 & 0.143 & 0.003 & 147.909 & 84.029 & 0.077 & 0.079 \\
\hline Sodium (mmol/L) & -35.397 & 20.269 & -0.083 & 0.081 & & & & \\
\hline Potassium (mmol/L) & 332.059 & 80.169 & 0.194 & $<0.001$ & 116.298 & 73.740 & 0.068 & 0.116 \\
\hline Total cholesterol (mg/dL) & -5.400 & 1.127 & -4.790 & $<0.001$ & -6.367 & 4.268 & -0.261 & 0.137 \\
\hline HDL cholesterol (mg/dL) & -6.765 & 3.188 & -0.101 & 0.034 & 1.431 & 4.013 & 0.021 & 0.722 \\
\hline LDL cholesterol (mg/dL) & -5.445 & 1.272 & -0.200 & $<0.001$ & 4.346 & 4.729 & 0.159 & 0.359 \\
\hline Triglyceride (mg/dL) & -0.531 & 0.843 & -0.030 & 0.529 & & & & \\
\hline Hemoglobin $(\mathrm{g} / \mathrm{dL})$ & -111.670 & 29.536 & -0.177 & $<0.001$ & -68.040 & 27.011 & -0.108 & 0.012 \\
\hline $25(\mathrm{OH})$ vit $\mathrm{D}(\mathrm{nmol} / \mathrm{L})$ & 10.942 & 5.081 & 0.102 & 0.032 & 4.772 & 4.565 & 0.045 & 0.297 \\
\hline hs-CRP (mg/dL) & 35.110 & 27.009 & 0.063 & 0.194 & & & & \\
\hline Proteinuria & $1,177.75$ & 301.672 & 0.183 & $<0.001$ & 539.393 & 267.913 & 0.084 & 0.045 \\
\hline Hematuria & 98.655 & 98.050 & 0.048 & 0.315 & & & & \\
\hline
\end{tabular}

GDF-15, growth differentiation factor-15; BMI, body mass index; eGFR, estimated glomerular filtration rate; HDL, high-density lipoprotein; LDL, low-density lipoprotein; 25(OH) vit D, 25-hydroxy vitamin D; hs-CRP, high-sensitivity C-reactive protein.

\section{Measurement of Plasma GDF-15}

Venous blood samples were obtained from the participants in the morning under fasting condition. Plasma was separated by centrifugation and stored at $-80^{\circ} \mathrm{C}$ until analysis. Plasma GDF-15 was measured using a human GDF-15 enzyme-linked immunosorbent assay (ELISA) kit (Cohesion Biosciences, London, UK), according to the manufacturer's instructions. All measurements were performed by investigators blinded to individual participant data.

\section{Statistics}

Continuous variables are presented as the mean \pm standard deviation (SD), and categorical data are reported as the frequency and percentage. An analysis of continuous variables was performed using Student's $t$ test, and that of categorical variables used the Chisquare or Fisher's exact test, as appropriate. Correlations between plasma GDF-15 level and clinical and biochemical parameters were investigated by performing linear and multiple regression analyses. Receiver operating characteristic (ROC) curves were drawn to determine the sensitivity, specificity, and area under the curve (AUC) by which CKD was predicted. A $p$ value $<0.05$ was considered to be statistically significant. All statistical analyses were carried out using the SPSS software version 19.0 (SPSS Inc., Chicago, IL, USA). 
Fig. 1. Association between plasma GDF-15 level and eGFR. GDF15 , growth differentiation factor-15; eGFR, estimated glomerular filtration rate.

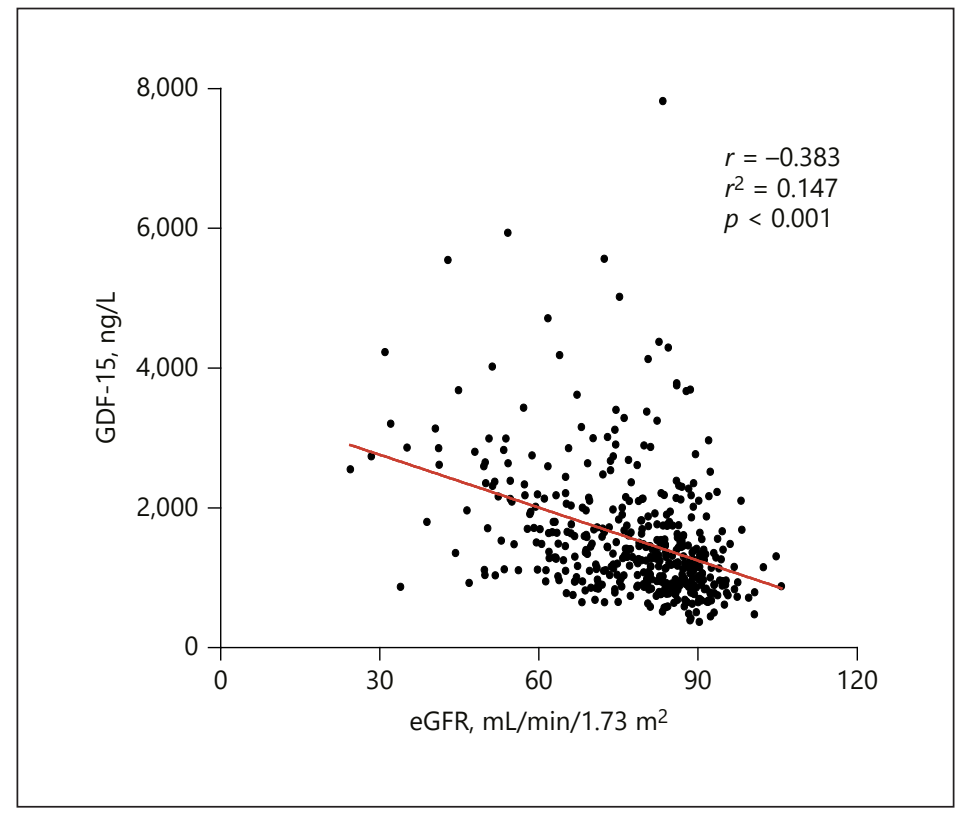

\section{Results}

\section{Study Population Characteristics}

The clinical and biochemical characteristics of the 443 participants are shown in Table 1. The mean age of the participants was $76.49 \pm 3.91$ years and $229(51.7 \%)$ subjects were male. Of the participants, 241 (54.4\%) had hypertension, and 88 (19.9\%) had DM. The mean creatinine level was $0.84 \pm 0.25 \mathrm{mg} / \mathrm{dL}$, with a mean eGFR of $77.74 \pm 13.83 \mathrm{~mL} / \mathrm{min} / 1.73 \mathrm{~m}^{2}$. Proteinuria was observed in $9(2.0 \%)$ participants, and hematuria was observed in 117 (26.4\%) participants. The mean GDF-15 level was 1,556.32 $\pm 910.09 \mathrm{ng} / \mathrm{L}$.

\section{Associations between GDF-15 and Clinical and Biochemical Parameters}

Table 2 shows the correlation between plasma GDF-15 levels and clinical and biochemical parameters. In a simple regression analysis, GDF-15 levels were negatively correlated with eGFR ( $r=-0.383, p<0.001$, Table 2 and Fig. 1). GDF-15 levels also tended toward negative significance with regard to total cholesterol, high-density lipoprotein (HDL) cholesterol, and hemoglobin, but toward positive significance with age, proteinuria, dyslipidemia, DM, coronary artery disease, protein, glucose, calcium, phosphorus, potassium, and 25-hydroxy vitamin D. In multiple linear regression analysis, eGFR $(r=-0.259, p<0.001)$, proteinuria $(r=0.084, p=0.045)$, DM $(r=0.357, p<0.001)$, and hemoglobin $(r=-0.107, p<0.013)$ were found to correlate significantly with GDF-15.

\section{CKD and GDF-15}

To analyze whether plasma GDF-15 can discern the presence of renal dysfunction in the elderly, we divided the study population into two groups: CKD and non-CKD. The characteristics of the two groups are summarized in Table 3. Participants in the CKD group were likely to be older than those in the non-CKD group (77.67 \pm 4.09 and $76.34 \pm 3.87$, respectively, $p=$ 0.022). The CKD group had significantly higher levels of potassium and lower levels of LDL cholesterol, triglyceride, and hemoglobin. Plasma GDF-15 was significantly higher in the CKD group than in the non-CKD group (Table 3 and Fig. 2, $p<0.001$ ). As shown in Figure 3, we 
Fig. 2. Plasma level of GDF-15 in elderly with or without CKD. ${ }^{*} p<$ 0.001 . GDF-15, growth differentiation factor-15; CKD, chronic kidney disease.

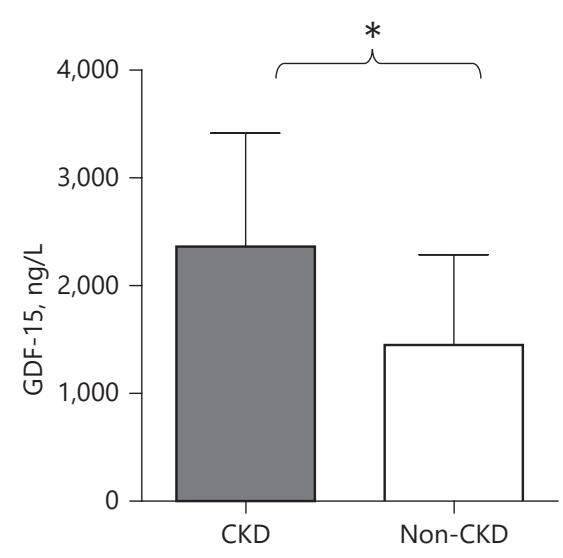

Table 3. Characteristics of the study population with or without CKD

\begin{tabular}{lccr}
\hline & CKD $(n=51)$ & Non-CKD $(n=392)$ & $p$ \\
\hline Age, years & $77.67 \pm 4.09$ & $76.34 \pm 3.87$ & 0.022 \\
Gender (M:F) & $30: 21$ & $199: 193$ & 0.279 \\
BMI & $24.96 \pm 3.62$ & $24.17 \pm 2.76$ & 0.138 \\
Hypertension, $n$ (\%) & $34(66.7 \%)$ & $207(52.8 \%)$ & 0.062 \\
Dyslipidemia, $n$ (\%) & $18(35.3 \%)$ & $114(29.5 \%)$ & 0.400 \\
Diabetes mellitus, $n(\%)$ & $15(30 \%)$ & $73(18.6 \%)$ & 0.058 \\
Protein, g/dL & $6.98 \pm 4.01$ & $7.01 \pm 0.38$ & 0.539 \\
Albumin, g/dL & $4.29 \pm 0.29$ & $4.32 \pm 0.23$ & 0.391 \\
Creatinine, mg/dL & $1.28 \pm 0.30$ & $0.78 \pm 0.16$ & $<0.001$ \\
eGFR, mL/min/1.73 m 2 & $81.40 \pm 9.45$ & $<0.001$ \\
Glucose, mg/dL & $49.58 \pm 0.30$ & $103.09 \pm 18.39$ & 0.167 \\
Calcium, mg/dL & $108.92 \pm 29.03$ & $9.24 \pm 0.36$ & 0.233 \\
Phosphorus, mg/dL & $9.31 \pm 0.36$ & $3.49 \pm 0.45$ & 0.231 \\
Sodium, mmol/L & $3.60 \pm 0.60$ & $141.30 \pm 2.17$ & 0.617 \\
Potassium, mmol/L & $141.14 \pm 1.81$ & $4.47 \pm 0.53$ & 0.001 \\
Total cholesterol, mg/dL & $4.73 \pm 0.44$ & $186.81 \pm 38.04$ & 0.015 \\
HDL cholesterol, mg/dL & $173.20 \pm 30.53$ & $52.51 \pm 13.50$ & 0.070 \\
LDL cholesterol, mg/dL & $48.86 \pm 13.38$ & $111.68 \pm 33.70$ & 0.008 \\
Triglyceride, mg/dL & $98.49 \pm 28.47$ & $113.17 \pm 50.30$ & 0.035 \\
Hemoglobin, g/dL & $129.25 \pm 57.79$ & $13.45 \pm 1.40$ & 0.009 \\
25(OH) vit D, nmol/L & $12.80 \pm 1.62$ & $21.01 \pm 8.29$ & 0.026 \\
hs-CRP, mg/dL & $24.18 \pm 9.44$ & $1.23 \pm 1.49$ & 0.213 \\
GDF-15, ng/L & $1.66 \pm 2.29$ & $1,451.23 \pm 835.79$ & $<0.001$ \\
Proteinuria, $n$ (\%) & $4 \%(1.0 \%)$ & 0.001 \\
Hematuria, $n$ (\%) & $11(21.6 \%)$ & $106(27.0 \%)$ & 0.257 \\
\hline
\end{tabular}

CKD, chronic kidney disease; BMI, body mass index; eGFR, estimated glomerular filtration rate; HDL, highdensity lipoprotein; LDL, low-density lipoprotein; 25(OH) vit D, 25-hydroxy vitamin D; hs-CRP, highsensitivity C-reactive protein; GDF-15, growth differentiation factor-15.

drew an ROC curve to determine the AUC of GDF-15 levels and to identify an optimal cut-off value for discrimination between the elderly with or without CKD. The GDF-15 AUC was $0.793 \pm 0.033$ ( $95 \%$ CI $0.729-0.857, p<0.001$ ). The optimal GDF- 15 cut-off value that detects the presence of CKD was $1,699.4 \mathrm{ng} / \mathrm{L}$ (76.5\% sensitivity and $76.0 \%$ specificity). 
Kidney
Blood Pressure

Research

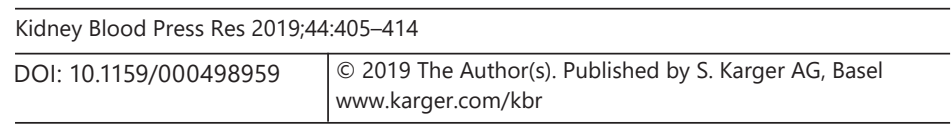

Kim et al.: Association between Plasma GDF-15 Levels and Renal Function in the Elderly

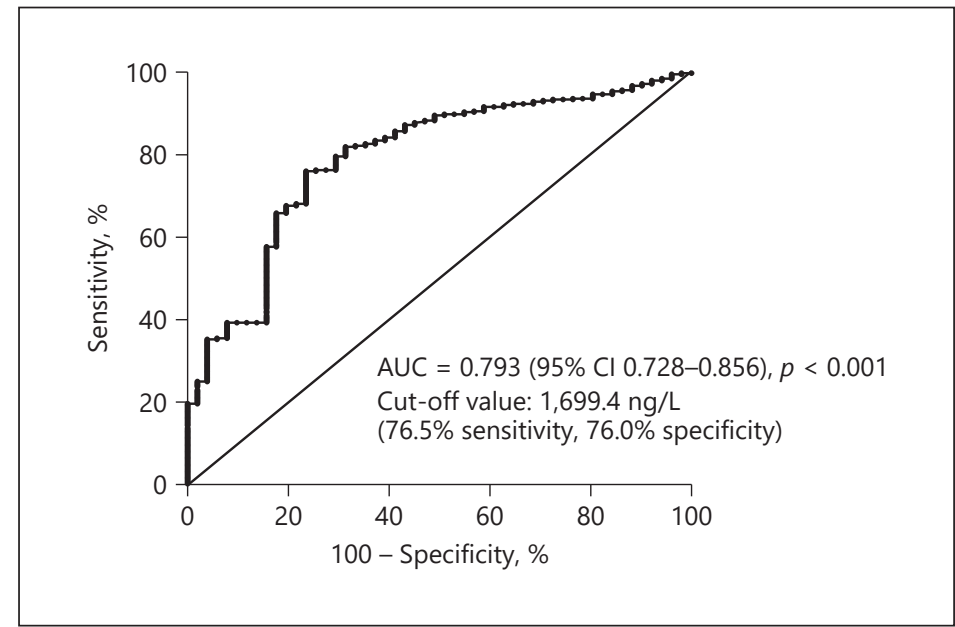

Fig. 3. ROC curves of GDF-15 for CKD in the elderly. ROC, receiver operating characteristic; GDF-15, growth differentiation factor-15; CKD, chronic kidney disease; AUC, area under the curve.

\section{Discussion}

In the present study, we investigated the association between plasma GDF-15 levels and renal function in the elderly using data from the KFACS. Our three principal findings are that, in the elderly: (1) GDF-15 is independently correlated with eGFR even after adjusting for other parameters; (2) GDF-15 correlates positively with proteinuria and DM, and negatively with hemoglobin level; and (3) increased GDF-15 was significantly associated with the presence of CKD.

GDF-15 has been indicated as a useful diagnostic and prognostic marker for cardiovascular diseases $[17,18]$, and studies based on GDF-15 are also being conducted in other disease fields, such as metabolic disorders, chronic inflammatory diseases, and malignancies $[7,19]$. In our study, GDF-15 correlated independently with DM and hemoglobin levels, findings that are consistent with previous studies [19-22]. Kempf et al. [19] reported that GDF-15 is related to insulin resistance and independently associated with the future development of abnormal glucose control. Other study groups have revealed that elevated GDF-15 in obese patients is further increased by the concurrence of type 2 DM [20]. An association between anemia and GDF-15 has also been documented in previous studies. Hong et al. [21] determined that anemia in type $2 \mathrm{DM}$ without overt renal dysfunction was independently associated with increasing hepcidin and GDF-15 levels. Another study revealed an association among GDF-15, anemia, and inflammation in elderly patients with early CKD [22].

Recently, GDF-15 level was found to have significant clinical association with renal disorders. Na et al. [11] demonstrated that plasma GDF-15 has an inverse correlation with eGFR and is associated with poor renal outcomes in patients with IgA nephropathy, and Ham et al. [10] reported similar trends in patients with idiopathic membranous nephropathy. Higher levels of GDF-15 predicted faster decline of renal function in patients with type 1 DM who have nephropathy [8] and starting dialysis in light chain amyloidosis patients [23]. Viji et al. [12] demonstrated that the level of plasma GDF-15 strongly correlates with intrarenal expression of GDF-15 mRNA, and that elevated GDF-15 was significantly associated with increased risk of renal function deterioration in patients with CKD.

Although several kidney biomarkers such as cystatin $\mathrm{C}$ and neutrophil gelatinase-associated lipocalin (NGAL) have been introduced, identification of a new marker is needed to assess accurate renal function and to understand the complex pathophysiology of renal disorders $[12,24]$. Our findings suggest that GDF-15 may be a useful kidney biomarker, especially in the elderly. In this study, we demonstrated that plasma GDF-15 level has an inverse 
graded association with eGFR, and this association remained after adjustment for various factors including age. This indicates that GDF-15 correlates with eGFR in the elderly independently rather than in relation to aging. We also revealed that the level of plasma GDF-15 was independent of BMI or gender. Considering muscle mass changes with aging, this may be an advantage in assessing renal function in the elderly $[25,26]$. In addition, we showed a positive correlation between GDF-15 and proteinuria. Since proteinuria has been reported as a marker for renal injury and a risk factor for CKD progression [27], GDF-15 is a potential biomarker for renal dysfunction in the elderly. Whether the GDF-15 level is a risk factor for CKD progression in the elderly will be evaluated in our next study using follow-up data of the KFACS.

While the mechanisms by which increased GDF-15 level could reflect the presence of renal disorders and predict disease prognosis are still unclear, some proposals have been suggested. Previous studies have reported the increase of GDF-15 after organ injury, including kidney damage. Simonson et al. [9] have established that urinary levels of GDF-15 have a positive correlation with proximal tubule injury in a mouse model. Zimmers et al. [6] demonstrated induction of GDF-15 expression after kidney or lung injury in murine models and suggested that GDF-15 is an early mediator in organ injury. Abulizi et al. [28] have determined that GDF-15 deficiency in a lipopolysaccharide-induced renal injury model aggravates renal damage. Other investigators demonstrated that genetic deletion of GDF-15 exacerbated injury in the renal interstitium and tubules in diabetic mice [29]. Based on these findings, we deduce that GDF-15 expression increases in the relatively early stage of renal injury to protect from tissue damage and is therefore able to predict disease by comparing this increase to the degree of tissue injury. Further studies are needed to understand the exact underlying mechanisms involved.

Our study has some potential limitations. First, the participants enrolled in the present study were predominantly Korean. Therefore, the results should be generalized with caution, and further studies are needed to assess the clinical value of GDF-15 in different populations. Second, serum creatinine was measured only once, which could lead to misclassification of the study population and thus may not have allowed us to accurately evaluate AKI; note that we could not obtain additional data because of the features of the database. In our next study, we plan to eliminate these drawbacks using prospective KFACS cohort data collection. Third, the glomerular filtration rate was not measured directly, but estimated using the CKD-EPI equation. This could lead to an over- or underestimation of renal function. Fourth, this was a cross-sectional study and could not evaluate the short-term or long-term prognostic value of GDF-15. However, considering that the KFACS is a longitudinal cohort study, we will be able to assess GDF-15 as a prognostic biomarker in our next study. Finally, we could not determine the underlying mechanisms of our findings. Further longitudinal and comparative studies are needed to investigate the mechanism responsible for the association between GDF-15 and renal function in the elderly. However, our study is significant because it is the first to establish a significant correlation between plasma GDF-15 and eGFR in a well-characterized cohort that represented the general aged population and is the first to analyze its optimal cut-off level for discriminating the presence of renal dysfunction in the elderly. Our findings could be an opportunity to identify a new biomarker for renal dysfunction in elderly individuals and may lead to further studies to understand the underlying pathophysiology of renal dysfunction and GDF-15 in the elderly.

In conclusion, plasma GDF-15 has a negative correlation with eGFR in the elderly and is significantly elevated in elderly participants with CKD. Our results indicate that GDF-15 could be a potential clinical parameter for detecting renal dysfunction in the elderly. Further longitudinal, comparative, and multiethnic studies are needed to ascertain the underlying mechanisms. 


\section{Kidney Blood Pressure Research}

\begin{tabular}{l|l}
\hline Kidney Blood Press Res 2019;44:405-414 \\
\hline DOI: 10.1159/000498959 & $\begin{array}{l}\text { ○ 2019 The Author(s). Published by S. Karger AG, Basel } \\
\text { www.karger.com/kbr }\end{array}$ \\
\hline
\end{tabular}

Kim et al.: Association between Plasma GDF-15 Levels and Renal Function in the Elderly

\section{Acknowledgements}

The authors thank the Korean Frailty and Aging Cohort Study Group. This research was supported by a grant of the Korea Health Technology R\&D Project through the Korean Health Industry Development Institute (KHIDI), funded by the Ministry of Health and Welfare, Republic of Korea (grant number: HI15C3153).

\section{Disclosure Statement}

The authors declare that there are no conflicts of interests.

\section{References}

1 Anderson S, Halter JB, Hazzard WR, Himmelfarb J, Horne FM, Kaysen GA, et al.; workshop participants. Prediction, progression, and outcomes of chronic kidney disease in older adults. J Am Soc Nephrol. 2009 Jun; 20(6):1199-209.

2 Mallappallil M, Friedman EA, Delano BG, McFarlane SI, Salifu MO. Chronic kidney disease in the elderly: evaluation and management. Clin Pract (Lond). 2014;11(5):525-35.

3 Moranne 0, Fafin C, Roche S, Francois M, Allot V, Potier J, et al. Treatment plans and outcomes in elderly patients reaching advanced chronic kidney disease. Nephrol Dial Transplant. 2018 Dec;33(12):2182-91.

4 Heringlake M, Schön J, Paarmann H. The kidney in critical illness: how to monitor a pivotal organ system. Best Pract Res Clin Anaesthesiol. 2013 Jun;27(2):271-7.

5 Buendgens L, Yagmur E, Bruensing J, Herbers U, Baeck C, Trautwein C, et al. Growth Differentiation Factor-15 Is a Predictor of Mortality in Critically Ill Patients with Sepsis. Dis Markers. 2017;2017:5271203.

6 Zimmers TA, Jin X, Hsiao EC, McGrath SA, Esquela AF, Koniaris LG. Growth differentiation factor-15/macrophage inhibitory cytokine-1 induction after kidney and lung injury. Shock. 2005 Jun;23(6):543-8.

7 Adela R, Banerjee SK. GDF-15 as a Target and Biomarker for Diabetes and Cardiovascular Diseases: A Translational Prospective. J Diabetes Res. 2015;2015:490842.

8 Lajer M, Jorsal A, Tarnow L, Parving HH, Rossing P. Plasma growth differentiation factor-15 independently predicts all-cause and cardiovascular mortality as well as deterioration of kidney function in type 1 diabetic patients with nephropathy. Diabetes Care. 2010 Jul;33(7):1567-72.

9 Simonson MS, Tiktin M, Debanne SM, Rahman M, Berger B, Hricik D, et al. The renal transcriptome of db/db mice identifies putative urinary biomarker proteins in patients with type 2 diabetes: a pilot study. Am J Physiol Renal Physiol. 2012 Apr;302(7):F820-9.

10 Ham YR, Song CH, Bae HJ, Jeong JY, Yeo MK, Choi DE, et al. Growth Differentiation Factor-15 as a Predictor of Idiopathic Membranous Nephropathy Progression: A Retrospective Study. Dis Markers. 2018 Feb;2018: 1463940.

11 Na KR, Kim YH, Chung HK, Yeo MK, Ham YR, Jeong JY, et al. Growth differentiation factor 15 as a predictor of adverse renal outcomes in patients with immunoglobulin A nephropathy. Intern Med J. 2017 Dec;47(12): 1393-9.

12 Nair V, Robinson-Cohen C, Smith MR, Bellovich KA, Bhat ZY, Bobadilla M, et al. Growth Differentiation Factor-15 and Risk of CKD Progression. J Am Soc Nephrol. 2017 Jul;28(7):2233-40.

13 Breit SN, Carrero JJ, Tsai VW, Yagoutifam N, Luo W, Kuffner T, et al. Macrophage inhibitory cytokine-1 (MIC-1/ GDF15) and mortality in end-stage renal disease. Nephrol Dial Transplant. 2012 Jan;27(1):70-5.

14 Eggers KM, Kempf T, Wallentin L, Wollert KC, Lind L. Change in growth differentiation factor 15 concentrations over time independently predicts mortality in community-dwelling elderly individuals. Clin Chem. 2013 Jul;59(7):1091-8.

15 Won CW, Lee Y, Choi J, Kim KW, Park Y, Park H, et al. Starting construction of frailty cohort for elderly and intervention study. Ann Geriatr Med Res. 2016;20(3):114-7.

16 Levey AS, Stevens LA, Schmid CH, Zhang YL, Castro AF 3rd, Feldman HI, et al.; CKD-EPI (Chronic Kidney Disease Epidemiology Collaboration). A new equation to estimate glomerular filtration rate. Ann Intern Med. 2009 May;150(9):604-12.

17 Kempf T, von Haehling S, Peter T, Allhoff T, Cicoira M, Doehner W, et al. Prognostic utility of growth differentiation factor-15 in patients with chronic heart failure. J Am Coll Cardiol. 2007 Sep;50(11):1054-60.

18 Wollert KC, Kempf T, Wallentin L. Growth Differentiation Factor 15 as a Biomarker in Cardiovascular Disease Clin Chem. 2017 Jan;63(1):140-51.

19 Kempf T, Guba-Quint A, Torgerson J, Magnone MC, Haefliger C, Bobadilla M, et al. Growth differentiation factor 15 predicts future insulin resistance and impaired glucose control in obese nondiabetic individuals: results from the XENDOS trial. Eur J Endocrinol. 2012 Nov;167(5):671-8. 
20 Dostálová I, Roubícek T, Bártlová M, Mráz M, Lacinová Z, Haluzíková D, et al. Increased serum concentrations of macrophage inhibitory cytokine- 1 in patients with obesity and type 2 diabetes mellitus: the influence of very low calorie diet. Eur J Endocrinol. 2009 Sep;161(3):397-404.

21 Hong JH, Choi YK, Min BK, Park KS, Seong K, Lee IK, et al. Relationship between hepcidin and GDF15 in anemic patients with type 2 diabetes without overt renal impairment. Diabetes Res Clin Pract. 2015 Jul;109(1):64-70.

22 Lukaszyk E, Lukaszyk M, Koc-Zorawska E, Bodzenta-Lukaszyk A, Malyszko J. GDF-15, iron, and inflammation in early chronic kidney disease among elderly patients. Int Urol Nephrol. 2016 Jun;48(6):839-44.

23 Kastritis E, Papassotiriou I, Merlini G, Milani P, Terpos E, Basset M, et al. Growth differentiation factor-15 is a new biomarker for survival and renal outcomes in light chain amyloidosis. Blood. 2018 Apr;131(14):1568-75.

24 Alge JL, Arthur JM. Biomarkers of AKI: a review of mechanistic relevance and potential therapeutic implications. Clin J Am Soc Nephrol. 2015 Jan;10(1):147-55.

25 Fliser D. Assessment of renal function in elderly patients. Curr Opin Nephrol Hypertens. 2008 Nov;17(6) 604-8.

26 Raman M, Middleton RJ, Kalra PA, Green D. Estimating renal function in old people: an in-depth review. Int Urol Nephrol. 2017 Nov;49(11):1979-88.

27 Turin TC, James M, Ravani P, Tonelli M, Manns BJ, Quinn R, et al. Proteinuria and rate of change in kidney function in a community-based population. J Am Soc Nephrol. 2013 Oct;24(10):1661-7.

28 Abulizi P, Loganathan N, Zhao D, Mele T, Zhang Y, Zwiep T, et al. Growth Differentiation Factor-15 Deficiency Augments Inflammatory Response and Exacerbates Septic Heart and Renal Injury Induced by Lipopolysaccharide. Sci Rep. 2017 Apr;7(1):1037.

29 Mazagova M, Buikema H, van Buiten A, Duin M, Goris M, Sandovici M, et al. Genetic deletion of growth differentiation factor 15 augments renal damage in both type 1 and type 2 models of diabetes. Am J Physiol Renal Physiol. 2013 Nov;305(9):F1249-64. 